\title{
Suppression of midkine gene promotes the antitumoral effect of cisplatin on human gastric cancer cell line AGS in vitro and in vivo via the modulation of Notch signaling pathway
}

\author{
WENYAN TIAN, JIAQING SHEN and WEICHANG CHEN \\ The First Affiliated Hospital of Soochow University, Suzhou, Jiangsu 215006, P.R. China
}

Received September 4, 2016; Accepted May 30, 2017

DOI: $10.3892 /$ or.2017.5743

\begin{abstract}
Midkine (MK) is reported to be associated with the clinical stages and distant metastases in gastric cancer, and to positively regulate the proliferation of human gastric cancer cells. However, the possible mechanisms of MK in the development of gastric cancer are still not fully clarified. In this study, the therapeutic effect of MK inhibition in gastric cancer in vivo and in vitro was investigated, by knock-down of MK expression with a small interfering RNA (siRNA). MK was expressed in gastric carcinoma tissues and cancer cells. The cytotoxic effect of cisplatin on AGS cells in vitro was attenuated by recombinant human MK, but was promoted by suppressing MK expression via downregulating the Notch signaling pathway-related proteins (Notch 1, Notch 2, Delta-like 1 and Jagged 1). Suppression of MK expression also promoted the inhibitory effect of cisplatin on AGS cells in vivo. In concusion, suppression of midkine gene promoted the antitumoral effect of cisplatin on human gastric cell line AGS in vitro and in vivo via Notch signaling pathway.
\end{abstract}

\section{Introduction}

As one of the most common malignancies around the world, gastric cancer is a highly fatal disease and often diagnosed in an advanced state with scarce effective therapies. Despite the improvements in medical and surgical therapy during the past decades, the mortality and average 5-year survival rate have not obviously changed after successful surgical intervention (1-3). Therefore, an efficient therapy of this disease is extremely urgent, and new approaches, including gene therapy, are requied to improve treatment results (4).

Midkine (MK) is a heparin-binding growth factor, first discovered as a highly expressed gene $(M d k)$ during mouse embryogenesis (5). Although prominently expressed during

Correspondence to: Dr Weichang Chen, The First Affiliated Hospital of Soochow University, 188 Shi Zi Street, Suzhou, Jiangsu 215006, P.R. China

E-mail: weichangchen@126.com

Key words: midkine, small interfering RNA, gastric cancer cell-line AGS, cisplatin, Notch signaling pathway embryogenesis, MK is downregulated to neglible levels in healthy adults. Accumulating evidence indicates that MK is involved in numerous pathologies, including ischaemia, inflammation, autoimmunity and, most notably, in many cancers (6-11). As a soluble cytokine, the elevated MK is readily apparent in the blood and other body fluids, which makes it a relatively convenient, accessible, non-invasive and inexpensive biomarker for population screening and early disease detection. Several biological functions of MK are thought to contribute to tumorigenesis and tumor progression, which promotes the proliferation, survival, and tumorigenicity of different cells and stimulates angiogenesis $(12,13)$. MK is also thought to participate in the pathogenesis of gastric cancer and positively regulates the proliferation of human gastric cancer (14). However, the possible mechanisms of MK in the development of gastric cancer are still not fully clarified.

In this study, the therapeutic effect of MK inhibition in gastric cancer in vivo and in vitro was investigated, by knockdown of MK expression with a small interfering RNA (siRNA).

\section{Materials and methods}

Patients and tissue specimens. A total of 17 patients, who received surgery for gastric adenocarcinoma at the Department of General Surgery, the First Affiliated Hospital of Soochow University in 2015, were enrolled in the study. Complete clinical data and paraffin-embedded gastric cancer specimens were available for all patients. Gastric cancer patients were staged using the International Union Against Cancer (UICC) 1997 TNM staging criteria, and histological typing of the primary tumor was performed using the World Health Organization (WHO) criteria. Poorly differentiated $(n=5)$, moderately differentiated $(n=6)$ and well differentiated $(n=6)$ gastric adenocarcinoma was diagnosed. Five patients were diagnosed as poorly differentiated gastric adenocarcinoma, 6 as moderately differentiated and 6 as well differentiated. Prior to surgery, no patient received radiotherapy or chemotherapy. The use of the tissue samples was approved by the local Ethics Committee of the First Affiliated Hospital of Soochow University and the informed consent of the patients was obtained according to the institutional regulations.

Cell culture and transfection. Human pancreatic cancer cell lines GES-1, 803, SGC7901, MKN4 and AGS were kindly 
gifted by Laboratory of Cellular and Molecular Tumor Immunology of Soochow University, and were cultured in RPMI-1640 (Gibco, USA) supplemented with $10 \%$ fetal bovine serum (FBS), $100 \mathrm{U} / \mathrm{ml}$ penicillin and $100 \mu \mathrm{g} / \mathrm{ml}$ streptomycin in a humidified incubator at $37^{\circ} \mathrm{C}$ in $5 \% \mathrm{CO}_{2}$. The transfection procedure was as previously reported (14). The pLXSN or pLXSN-MK plasmid was transfected into packed GP293 cells with Lipofectamine $^{\mathrm{TM}} 2000$ reagent (Invitrogen, CA, USA). After $48 \mathrm{~h}, 1.5 \mathrm{ml}$ of virus supernatant from various plasmids was added to $80 \%$ confluent AGS cells, which were incubated at $37^{\circ} \mathrm{C}$ for $24 \mathrm{~h}$, and then screened with $\mathrm{G} 418(400 \mathrm{mg} / \mathrm{l})$. Monoclonal cells were selected and cultured further. The clones were screened for MK expression with western blot analysis. The nucleotide sequences of MK siRNA were 5'-GGAGCCGACUGCAAGUACATT-3' and 5'-UGUAC UUGCAGUCGGCUCCAA-3'. The negative control siRNA sequences were 5'-UUCUCCGAACGUGUCACGUTT-3' and 5'-ACGUGACACGUUCGGAGAATT-3'.

Cell viability assay. AGS cells were plated in $100 \mu 1$ medium per well in 96-well plates, blank and zero wells were set. One day after seeding, cell viability was measured with Cell Counting Kit-8 (Peptide Institute Inc., Osaka, Japan) at $48 \mathrm{~h}$ after transfection for 2-h culture at $37^{\circ} \mathrm{C}$, and the surival rate and inhibition rate were calculated. The $\mathrm{OD}$ value at the wavelength of $490 \mathrm{~nm}$ was detected using an enzyme-labeled analyzer. The cell survival rate was calculated based on the formula: the survival rate $=$ (the OD value of the experimental group/the OD value of the blank group) $x 100 \%$. For in vitro study, two experiments were carried out. In experiment 1 , the effect of recombinant human midkine (rhMK) (Abcam, UK) was tested. AGS cells were treated with negative control group, rhMK group $(5 \mu \mathrm{g} / \mathrm{ml})$, cisplatin group $(50 \mu \mathrm{g} / \mathrm{ml})$, cisplatin $(50 \mu \mathrm{g} / \mathrm{ml})+\operatorname{rhMK}$ group $(5 \mu \mathrm{g} / \mathrm{ml})$, cisplatin $(50 \mu \mathrm{g} / \mathrm{ml})+\gamma$-secretase inhibitor I (GSI; $1 \mu \mathrm{M})$, group and cisplatin $(50 \mu \mathrm{g} / \mathrm{ml})+\operatorname{rhMK}(5 \mu \mathrm{g} / \mathrm{ml})+\mathrm{GSI} \mathrm{I}$ $(1 \mu \mathrm{M})$ group. In experiment 2 , the effect of MK siRNA was tested. AGS cells were treated with negative control group, non-targeted siRNA group, MK siRNA group, cisplatin group $(50 \mu \mathrm{g} / \mathrm{ml})$, cisplatin group $(50 \mu \mathrm{g} / \mathrm{ml})+$ non-targeted siRNA group, and cisplatin group $(50 \mu \mathrm{g} / \mathrm{ml})+\mathrm{MK}$ siRNA group.

Annexin V/PI assay. AGS cells were plated in 6-well plate and treated as indicated above. After 48-h incubation cells were collected, washed in cold PBS twice and then mixed in $100 \mu \mathrm{l}$ of $1 \mathrm{X}$ binding buffer and incubated with an Annexin V/PI double staining solution ( $5 \mu 1$ FITC Annexin V and $5 \mu 1$ PI) (Sigma-Aldrich, St. Louis, MO, USA) at room temperature for $15 \mathrm{~min}$ (the cell density was adjusted to $1 \times 10^{6} / \mathrm{ml}$ ). The stained cells were analyzed by flow cytometry and the percentage of apoptotic cells were calculated with ModFitLT software (Verity Software House, Topsham, ME, USA). The percentage of apoptotic cells was calculated.

Western blot assay. Total proteins were prepared by standard procedures and quantified by the BCA method and loaded onto a $10 \%$ SDS-polyacrylamide gel. After electrophoresis, proteins were transferred onto a PVDF membrane by electroelution. The membrane was incubated with primary antibody (MK, Notch 1 and Notch 2) (Santa Cruz Biotechnology, Santa
Cruz, CA, USA) overnight at $4^{\circ} \mathrm{C}$. The next day, the membrane was washed and incubated with HRP-conjugated secondary antibodies (Santa Cruz Biotechnology) for $1 \mathrm{~h}$ at room temperature. After washing, the membrane was developed using the ECL-detection system, quickly dried, and exposed to ECL film. We also used anti- $\beta$-actin antibody (Santa Cruz Biotechnology) as an internal standard.

Immunohistochemical staining. The primary antibodies (MK, Delta-like 1 and PCNA) (Santa Cruz Biotechnology) were utilized for immunohistochemistry. After deparaffinization, endogenous peroxidase activity was blocked by incubating the $5-\mu \mathrm{m}$ tissue sections for $30 \mathrm{~min}$ with $0.3 \%$ hydrogen peroxide in methanol. The tissue sections were incubated with the primary antibodies in phosphate-buffered saline (PBS) containing $5 \%$ bovine serum albumin overnight at $4{ }^{\circ} \mathrm{C}$. After washing, the sections were stained with biotin-conjugated secondary antibodies and followed by horseradish peroxidase-conjugated streptavidin (Boster Biological Technology, Wuhan, China) for $30 \mathrm{~min}$. Diaminobenzidine (DAB) was used as the immunodetection substrate. The in situ cell death detection kit for TUNEL assay (Beyotime, China) was applied for analyzing cell death.

Immunofluorescence staining. Paraffin-embedded samples were deparaffinized and rehydrated. Sections were microwave treated (565 min) in EDTA buffer ( $\mathrm{pH}$ 9.0), allowed to cool for $30 \mathrm{~min}$, and washed in PBS. After being blocked for $20 \mathrm{~min}$ with $5 \%$ bovine serum albumin, slides were incubated overnight at $4^{\circ} \mathrm{C}$ with antibodies against Delta-like 1 (1:200 dilution) and against Jagged 1 (1:200 dilution). Sections were then rinsed in PBST (PBS+0.1\% Tween-20) and immunoreactive protein was detected using a secondary antibody (1:400 dilution) conjugated with fluorochrome Cy3 (Jackson ImmunoResearch Laboratory, USA) and a secondary antibody (1:400 dilution) conjugated with fluorochrome Alexa FluorH 488 (Jackson ImmunoResearch Laboratory, USA) for $1 \mathrm{~h}$ in the dark. After being rinsed in PBST, slides were mounted with Fluoromount $^{\mathrm{TM}}$ mounting medium (Sigma-Aldrich) with 4',6-diamidino-2-phenylindole (DAPI) (1:1,000 dilution). Fluorescence analysis was performed by using a confocal laser scanning microscope (LSM 710; Zeiss, Germany) and Zen 2009 software (Carl-Zeiss, Jena, Germany).

In vivo experiment. Six-week-old female athymic nude mice were used in the experiment in vivo. AGS cells were injected subcutaneously into the right anterior armpit of nude mice to establish an animal model of transplanted tumors. AGS cells were resuspended in serum-free RPMI-1640. The cell suspension was then injected subcutaneously $\left(5 \times 10^{7}\right.$ cells; total volume $0.5 \mathrm{ml}$ ) into the nude mice. Then mice were divided into six groups: group 1 was injected with AGS cells; group 2 was injected with AGS cells stably transfected with pLXSNnon-targeted siRNA; group 3 was injected with AGS cells stably transfected with pLXSN-MKsiRNA; group 4 was injected with AGS cells treated with cisplatin; group 5 was injected with AGS cells stably transfected with pLXSN-non-targeted siRNA, then treated with cisplatin; group 6 was injected with AGS cells stably transfected with pLXSN-MKsiRNA, then treated with cisplatin (ten mice in each group). Five days after 
A

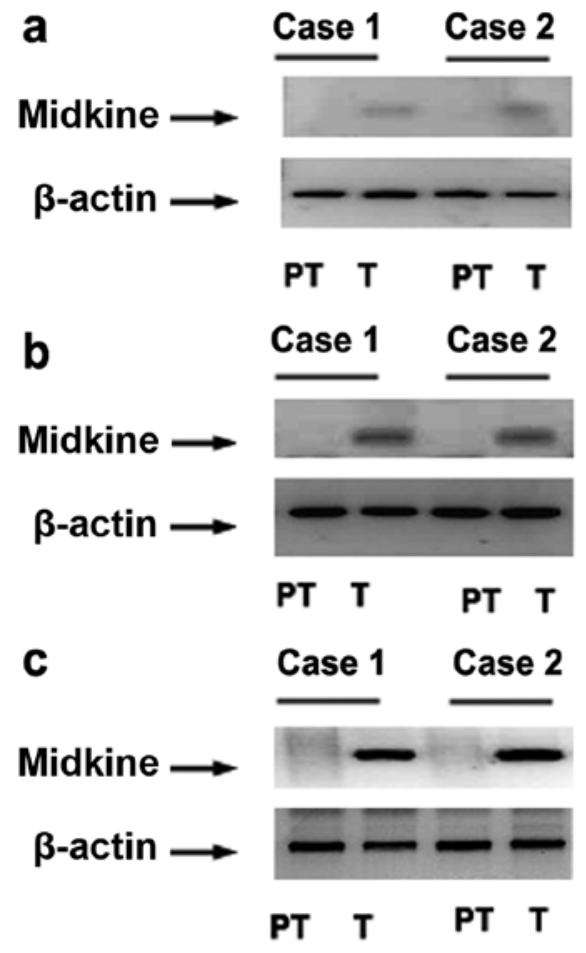

B

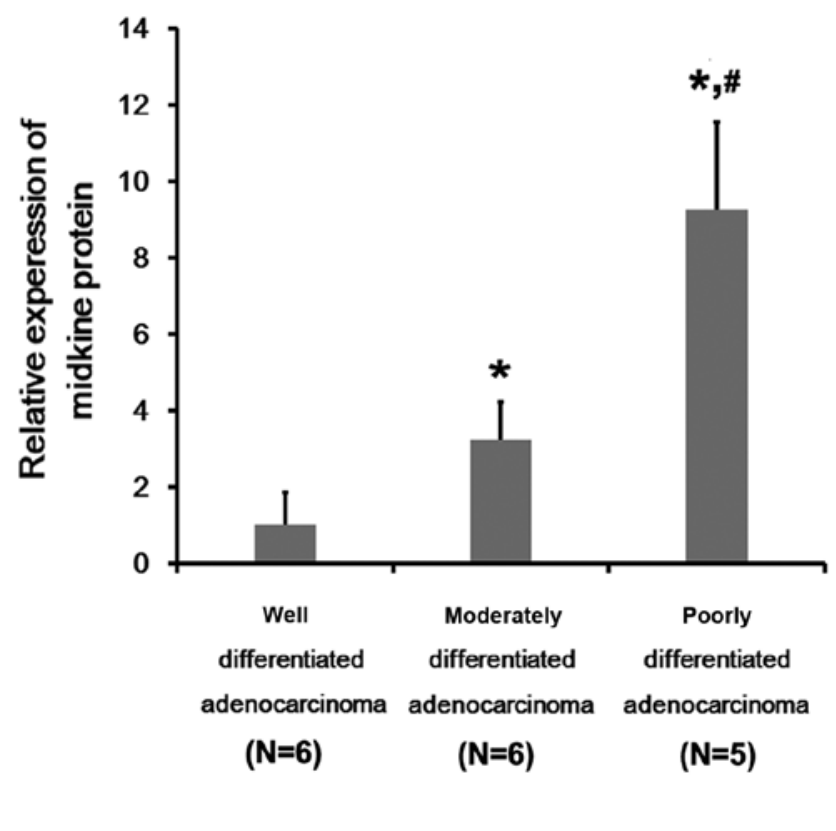

Figure 1. Expression of MK protein in gastric cancer tissues. (A) The different expression of MK protein in gastric cancer tissues by western blot analysis according to the histological differentiation. (a) Well differentiated; (b) moderately differentiated; (c) poorly differentiated. (B) The relative expression of MK protein. Data are expressed as mean \pm SE. ${ }^{p} \mathrm{p}<0.05$, versus well differentiated; ${ }^{*} \mathrm{p}<0.05$, versus moderately differentiated.

the transplantation, cisplatin $(2 \mathrm{mg} / \mathrm{kg})$ was administered peritoneally each day until the end of the study (15). Five weeks after the transplantation, mice were sacrificed and the tumor tissues were collected for histological examination and stored at $-80^{\circ} \mathrm{C}$ for protein extraction. The tumor size was measured every 7 days with calipers. The tumor volume was calculated with the formula: $\left(\mathrm{LxW}^{2}\right) / 2$, where $\mathrm{L}$ is the length and $\mathrm{W}$ the width of the tumor. After the mice were sacrificed, weights of the tumors were measured.

Statistical analysis. Data are expressed as mean \pm standard error (SE) and were analyzed using SPSS PC version 18.0 (SPSS Inc., Chicago, IL, USA). Statistical analysis was performed using one-analysis of variance (ANOVA) followed by SNK tests as post hoc test. Kruskal-Wallis test was used to evaluate the differences of categorical values followed by Mann-Whitney U tests as post hoc test. The criterion of significance was set as $\mathrm{p}$-value of $<0.05$.

\section{Results}

Expression of $M K$ in gastric cancer tissues and cancer cells. Seventeen patients were enrolled and gastric cancer tissues were collected. Of the patients 6 were diagnosed as well differentiated gastric adenocarcinoma, 6 as moderately differentiated and 5 as poorly differentiated. In well differentiated gastric adenocarcinoma, western blot analysis showed that MK protein was detectable in 4 patients, comapared with non-cancerous tissues adjacent to the cancer. The high expression of MK protein was found both in moderately differentiated and poorly differentiated adenocarcinoma. The relative expression of MK protein was upregulated in poorly differentiated tissues, nearly 3 -fold higher than that in the moderately differentiated tissues (Fig. 1). Next, we analyzed the location of MK in gastric cancer tissues. At the cellular level, the cytoplasm of the cancer cells was immunoreactive with anti-MK antibodies, as well as nucleolus immunoreactivity partly in poorly differentiated adenocarcinoma cases. However, fewer immunopositive cells were found in well differentiated tissues (Fig. 2). The expression of MK protein was also detected in gastric cell lines. The protein expression could be barely found in GES-1, 803 and SGC-7901. Higher expression of MK protein was detected in AGS cells, compared with MKN45 cells (Fig. 3). Therefore, AGS cells were used for further experiments.

RhMK attenuates the cytotoxic effect of cisplatin on AGS cells in vitro. We measured the survival and growth of AGS cells with or without rhMK treatment, using the CCK-8 kit assay. Compared with the NS control, rhMK could not affect the growth of AGS cells. Cisplatin obviously inhibited the proliferation and survival of AGS cells, while this inhibition was attenuated by rhMK treatment (Fig. 4). Annexin V/PI assay was applied to detect the cell death in vitro [Annexin V (+)/PI (-), early apoptosis; Annexin V (-)/PI (+), late apoptosis; Annexin V (-)/ PI (+), necrosis]. Results of Annexin V/PI assay showed that no obvious apoptosis or necrosis were found both in normal saline and rhMK-treated cells. In cisplatin-treated cells, significant apoptotic and necrotic cells were detected, and the necrosis was attenuated by rhMK treatment (Fig. 5). However, 


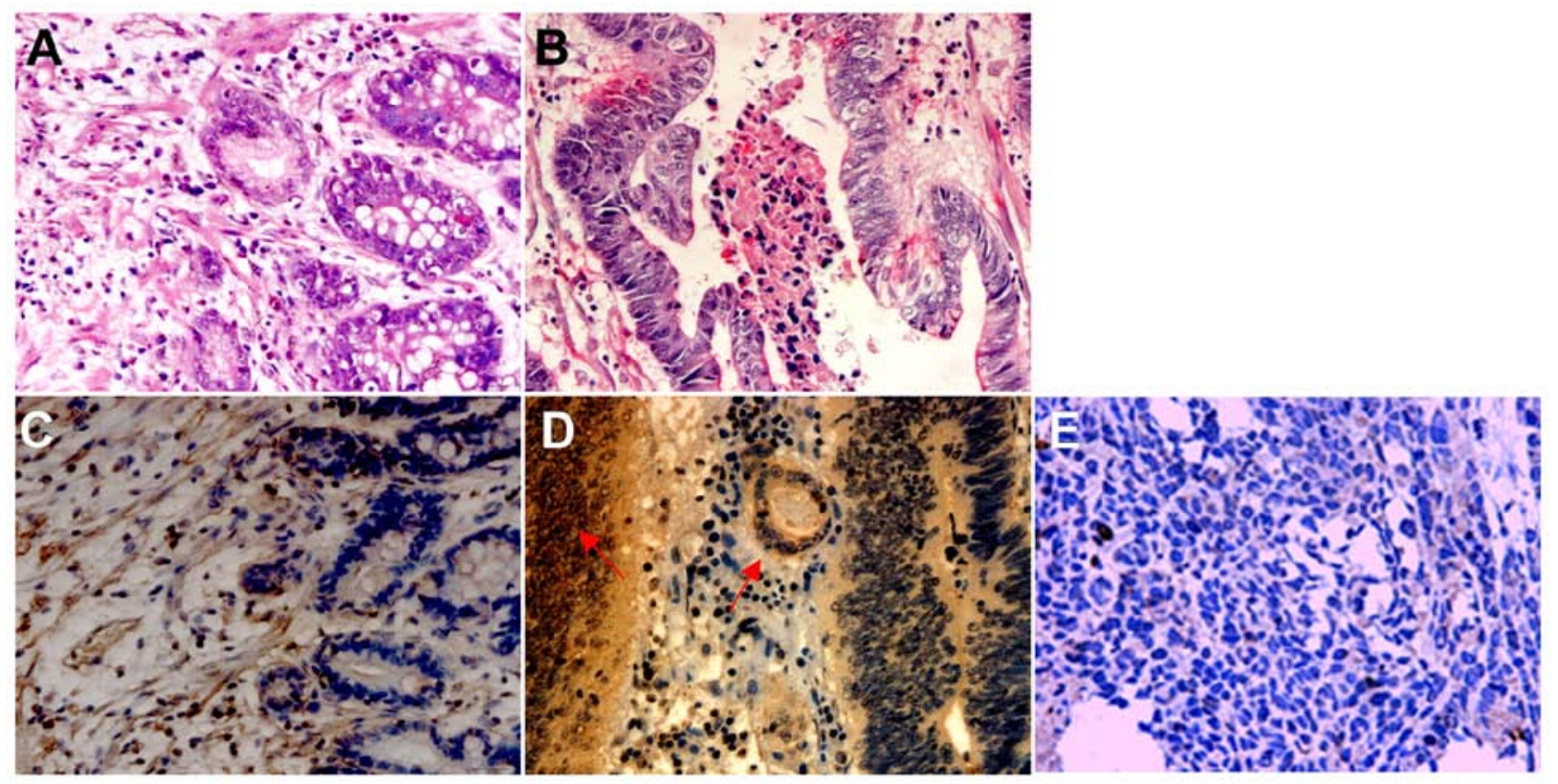

Figure 2. Representative images of H\&E-stained sections and immunohistochemical analysis of MK expression. (A and B) H\&E-stained sections of gastric adenocarcinoma (x200) [(A) well differentiated gastric adenocarcinoma; (B) poorly differentiated gastric adenocarcinoma]. (C and D) Immunohistochemically stained sections of MK expression (x200) [(C) well differentiated gastric adenocarcinoma; (D) poorly differentiated gastric adenocarcinoma]. Arrowheads indicate the cytoplasmic staining. (E) The negative control staining with control IgG.

A

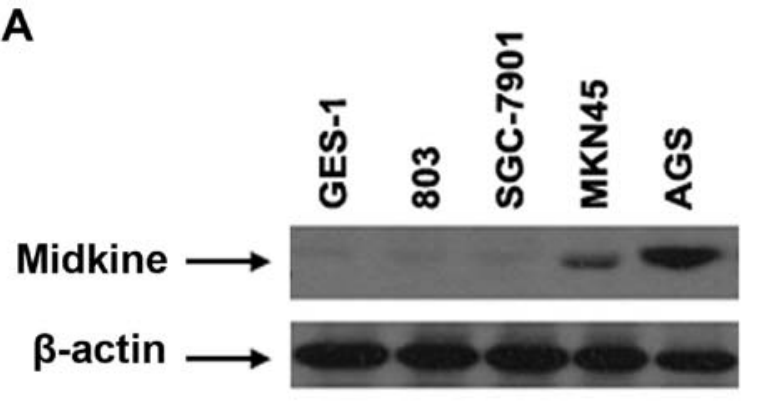

C

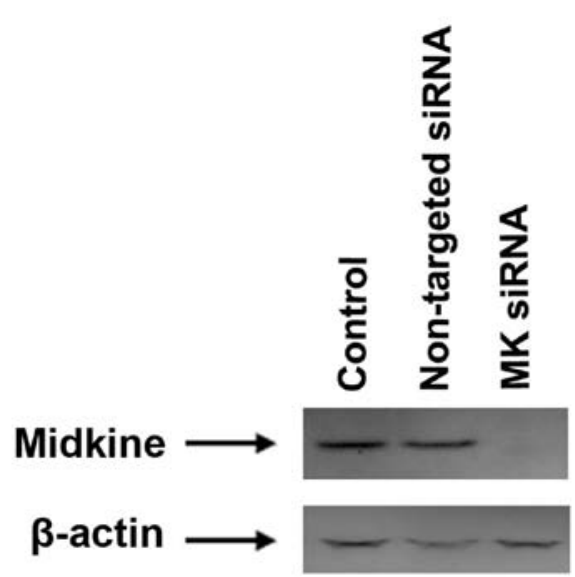

Figure 3. (A) Expression of MK protein in gastric cancer cell lines analyzed by western blot analysis. MK protein was barely found in GES-1, 803 and SGC-7901, but obviously detected in MKN45 and AGS. (B) The comparison between the concentration of MK in the supernatant in the medium and the concentration of recombinant MK by western blot analysis. (C) Expression of MK protein in AGS cells was significantly reduced by MK siRNA (20 nM, as previosly reported), compared with non-targeted siRNA.

the anti-proliferative effect of cisplatin on GES-1 cells was not influenced by rhMK treatment (Fig. 6). The expression of Delta and Notch can be affected by other cytotoxic drugs, according to previous reports, such as 5-FU, adriamycin, and doxorubicin $(16,17)$. By using $\gamma$-secretase inhibitor, the effects of cisplatin and rhMK were cancelled. 


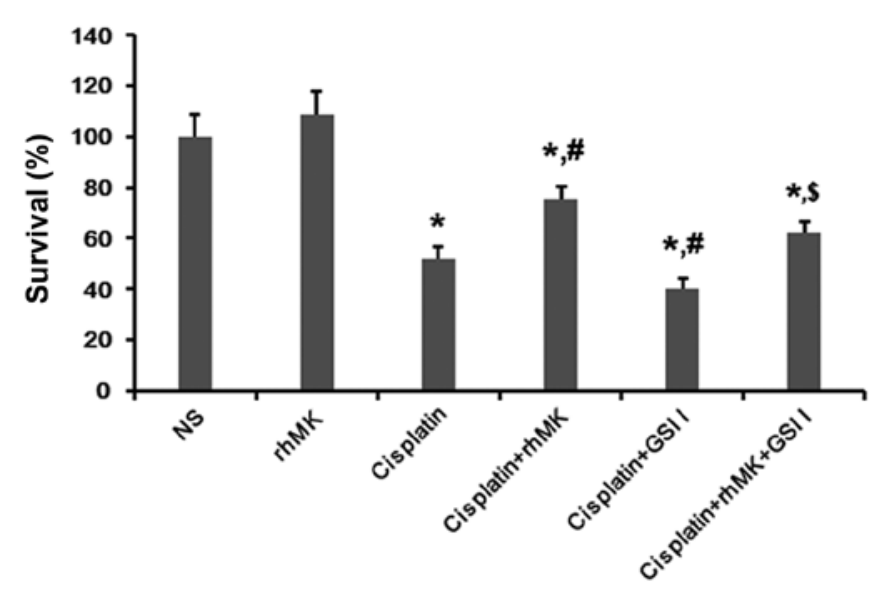

Figure 4. Inhibition of growth in AGS cells by cisplatin was attenuated by rhMK analyzed by CCK- 8 assay. Data were expressed as mean \pm SE " $<<0.05$, compared with normal saline-treated cells; ${ }^{*} \mathrm{p}<0.05$, compared with cisplatin-treated cells. ${ }^{\$} \mathrm{p}<0.05$, compared with cisplatin + rhMK-treated cells. rhMK, recombinant human midkine; GSI I, $\gamma$-secretase inhibitor I.

The cytotoxic effect of cisplatin on AGS cells is promoted by suppressing $M K$ expression in vitro. To determine whether the suppression of MK expression influenced the cell growth or not, MK-targeted siRNA was applied in this study. CCK-8 kit assay showed that, without cisplatin treatment, the proliferation and survival were not affected either by MK-siRNA or non-targeted siRNA, nor the percentage of apoptosis and necrosis in AGS cells by Annexin V/PI. In cisplatintreated cells, the growth was inhibited and the percentage of apoptosis and necrosis were obviously increased, and the apoptosis tended to be further increased by MK-siRNA, while non-targeted siRNA showed no such effect (Figs. 7 and 8).

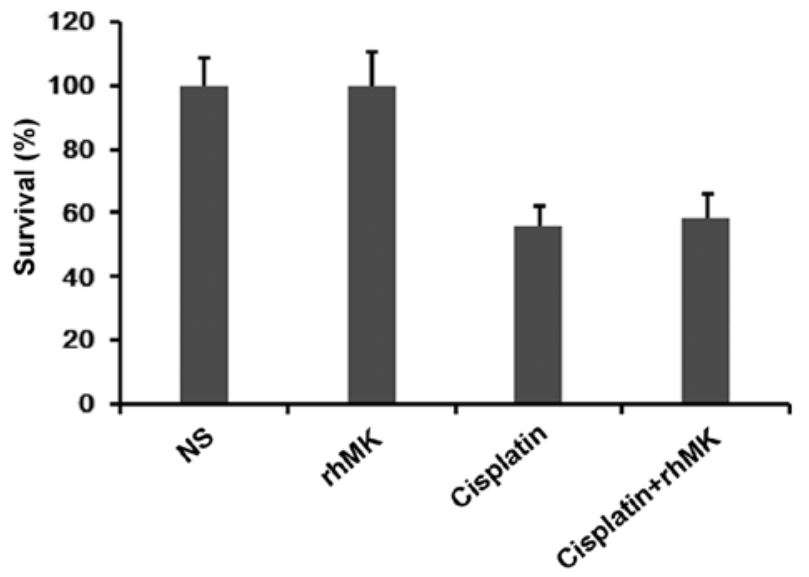

Figure 6. Effect of rhMK on the growth of GES-1 cells by cisplatin analyzed by CCK- 8 assay. Data are expressed as mean $\pm \mathrm{SE}$.

Immunofluorescent staining of Delta-like 1 and Jagged 1 was conducted to analyze the influence of the inhibition of MK expression upon the regulation of Notch signaling pathway related ligands. Without cisplatin intervention, non-targeted siRNA and MK-siRNA did not affect the expression of Delta-like 1 and Jagged 1, compared with NS-treated cells. After receiving cisplatin, the downregulation of Delta-like 1 and Jagged 1 protein expression could be clearly observed, and this change was enhanced by MK-siRNA. Western blot analysis showed that the expression of Notch 1 and Notch 2 was also reduced by MK-siRNA (Fig. 9).

Suppression of MK gene promotes the inhibitory effect of cisplatin on AGS cells in vivo. According to the results of the
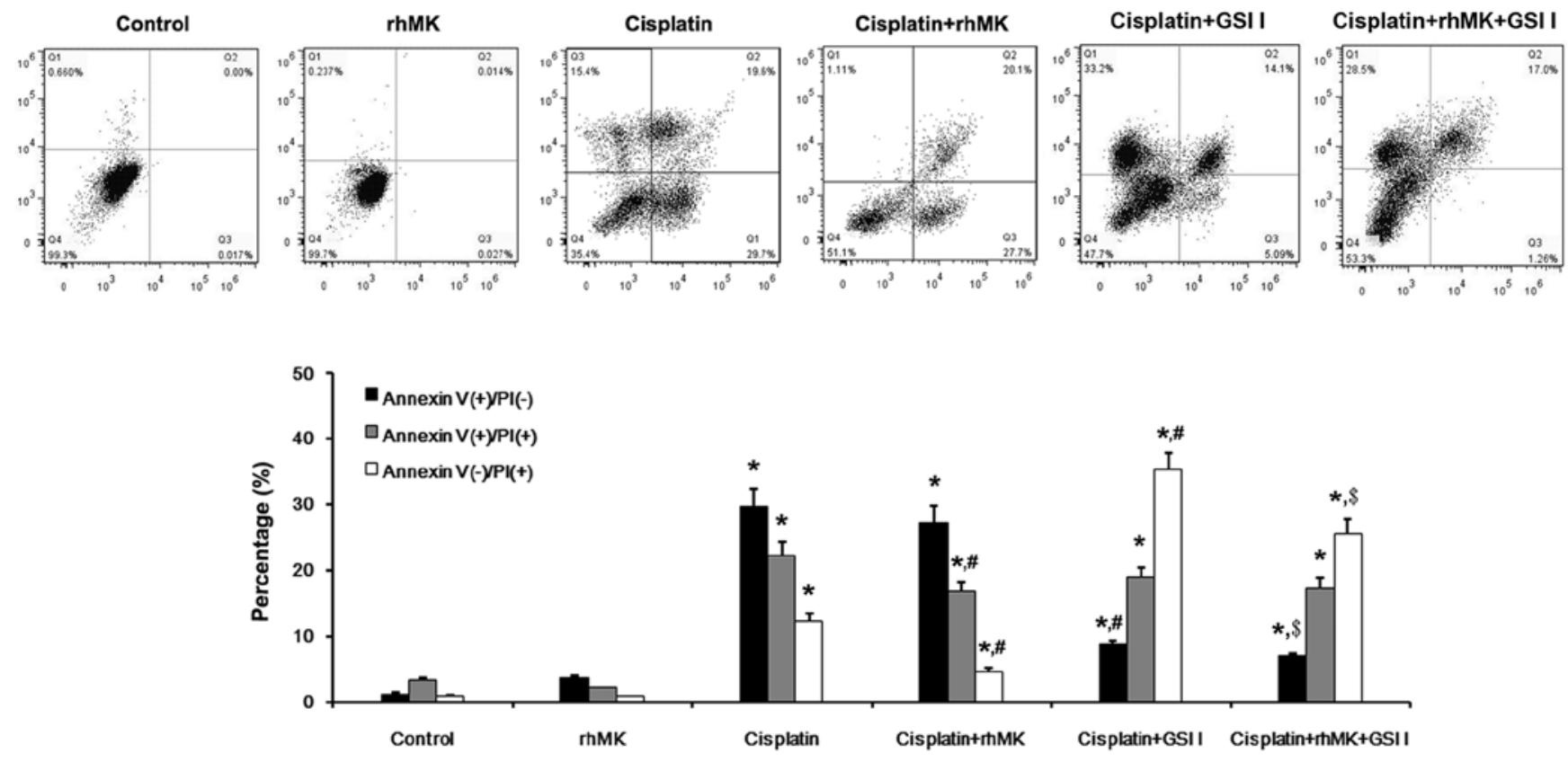

Figure 5. Impact of rhMK on cisplatin-induced cell death in AGS cells in vitro (Annexin V/PI assay). Significant apoptosis was detected in cisplatin-treated cells, which was attenuated by rhMK. Data are expressed as mean \pm SE. ${ }^{*} \mathrm{p}<0.05$, compared with normal saline-treated cells; ${ }^{*} \mathrm{p}<0.05$, compared with cisplatintreated cells. ${ }^{\$} \mathrm{p}<0.05$, compared with cisplatin + rhMK-treated cells. rhMK, recombinant human midkine; GSI I, $\gamma$-secretase inhibitor I. Annexin V (+)/ PI (-), early apoptosis; Annexin V (-)/PI (+), late apoptosis; Annexin V (-)/PI (+), necrosis. 


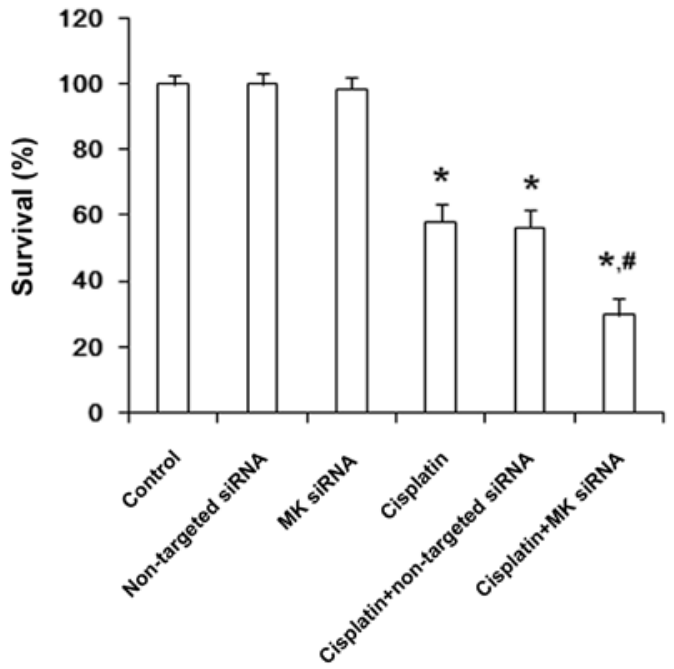

Figure 7. Impact of MK-targeted siRNA on cisplatin-induced growth inhibition in AGS cells in vitro (CCK-8 assay). Inhibitory effect of cisplatin on AGS cells was strengthened by MK siRNA. Data are expressed as mean $\pm \mathrm{SE}$. ${ }^{*} \mathrm{p}<0.05$, compared with normal saline-treated cells; ${ }^{*} \mathrm{p}<0.05$, compared with cisplatin-treated cells.

in vivo experiment, the treatment of cisplain alone exerted a beneficial effect on the gastric cancer in terms of the decreased tumor volume and weight, compared with the control groups. Furthermore, the tumor volume and weight were decreased more significantly after the combined treatment with cisplatin and MK-siRNA, during the whole observation period (Fig. 10). Histochemical staining indicated that the chemical density of Delta-like 1 and PCNA was reduced by cisplatin, which could be promoted by MK-siRNA. The TUNEL analysis revealed that cisplatin caused apoptosis in cancerous tissues was also enhanced by MK-siRNA (Fig. 11). The expression of Notch 1 and Notch 2 protein was also found to be reduced in cisplatintreated cells, and the reduction was promoted by MK-siRNA (Fig. 12).

\section{Discussion}

Gastric cancer is one of the most commonly seen malignancies with the highest mortality in China. Despite advances in its diagnosis and treatment, the prognosis for advanced gastric cancer is poor, with a 5-year survival rate of $<10 \%$. Therefore, it is crucial to develop new and more effective therapeutic strategies for this fatal disease. Numerous growth factors and their downstream signaling systems are involved in the development, progression, and dissemination of gastric cancer. Increased MK expression has been reported in various human carcinomas. As a heparin-binding growth factor, the expression of which is generally low or undetectable in adults, whereas it is high in various human cancers, including esophageal, gastric, urinary bladder, pancreatic, colorectal, breast, and lung carcinomas, neuroblastoma, and Wilms's tumor. Some reports
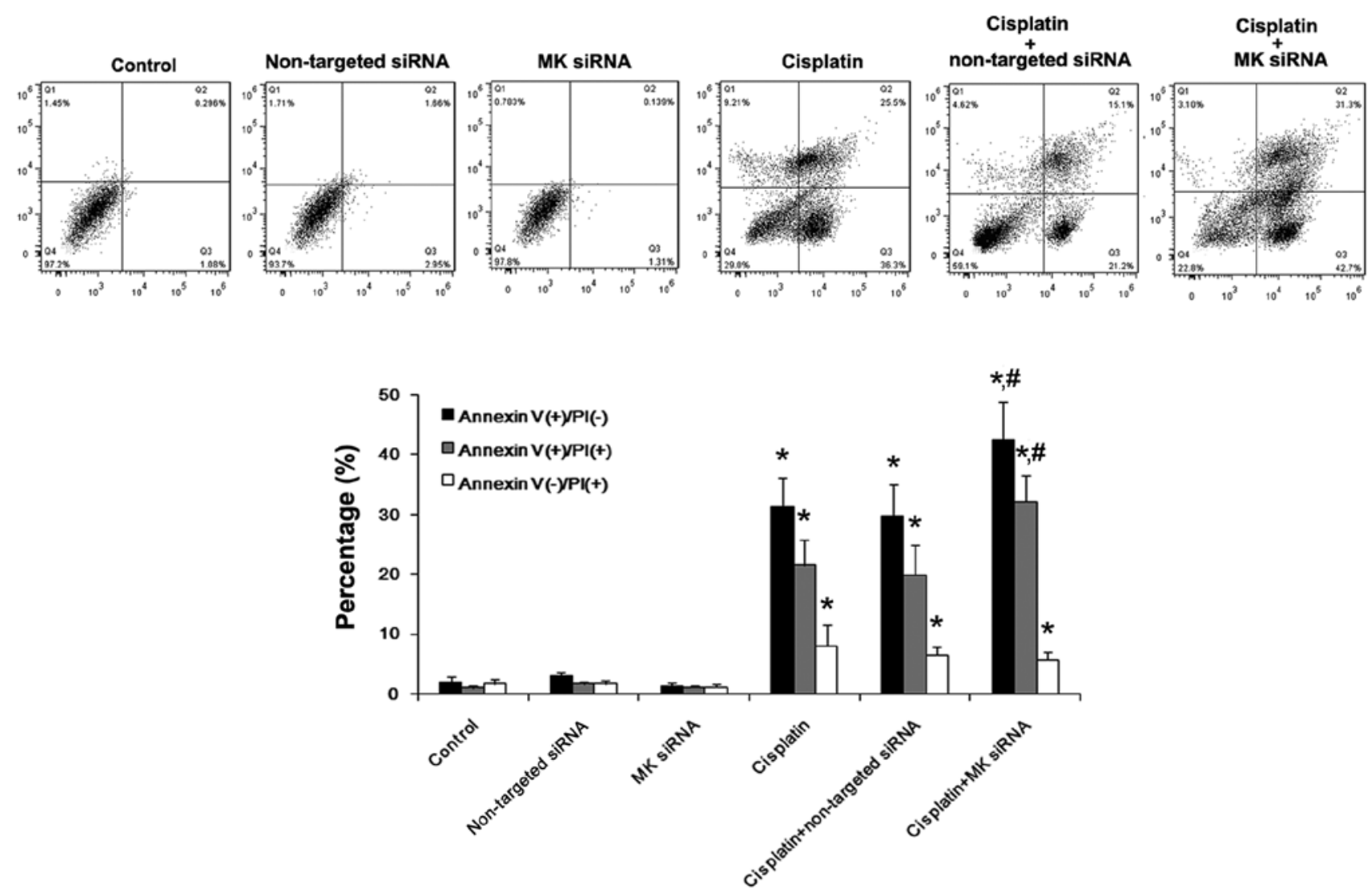

Figure 8. Impact of MK-targeted siRNA on cisplatin-induced cell death in AGS cells in vitro (Annexin V/PI assay). Significant apoptosis and necrosis were detected in cisplatin-treated cells. The apoptosis was further increased by MK siRNA. Data are expressed as mean \pm SE. "p $<0.05$, compared with normal saline-treated cells; ${ }^{*} \mathrm{p}<0.05$, compared with cisplatin-treated cells. Annexin V (+)/PI (-), early apoptosis; Annexin V (-)/PI (+), late apoptosis; Annexin V (-)/ PI (+), necrosis. 
A

Control Non-targeted siRNA MK siRNA Cisplatin

Cisplatin Cisplatin
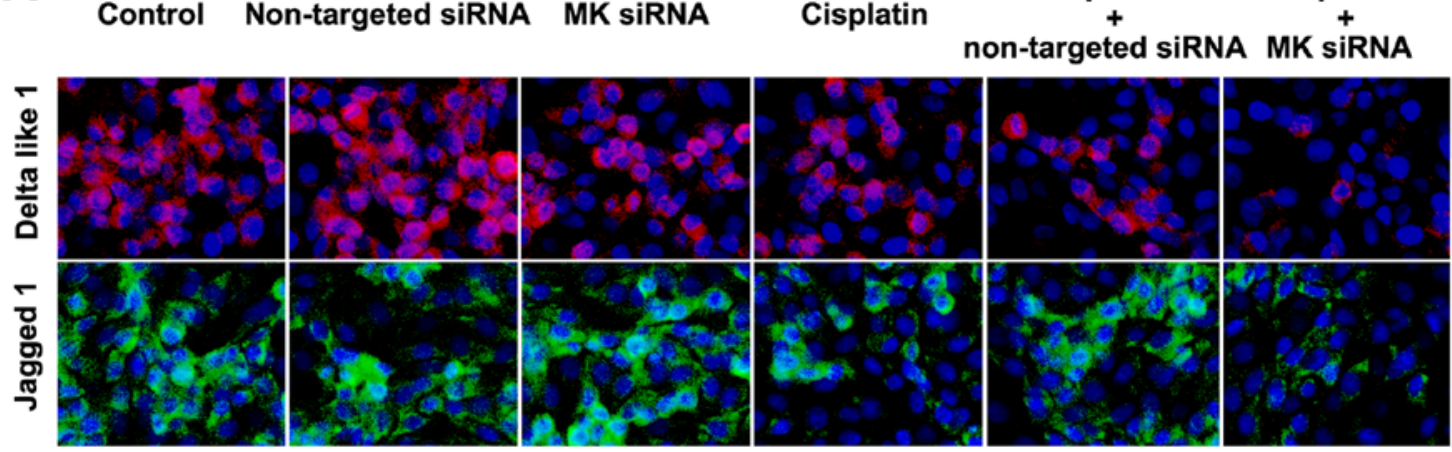

B

C
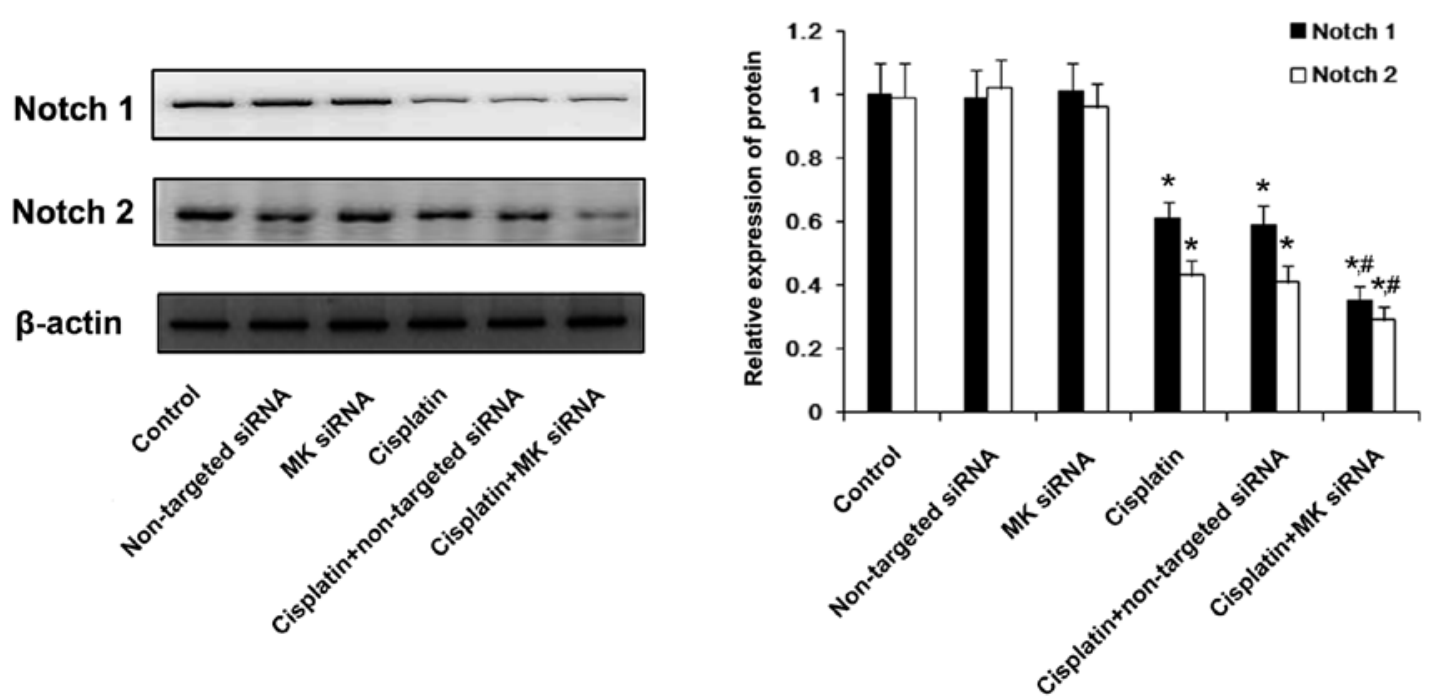

Figure 9. The effect of cisplatin and MK siRNA on the expression of Notch signaling pathway related proteins in AGS in vitro. (A) Immunofluorescent staining showed that cisplatin treatment could downregulate the expression of Delta-like 1 and Jagged 1, and this effect was strengthened by MK siRNA. (B) Western blot analysis showed that the downregulation of Notch 1 and Notch 2 induced by cisplatin was strengthened by MK siRNA. (C) The relative expression of Notch 1 and Notch 2 protein. Data are expressed as mean \pm SE. " $\mathrm{p}<0.05$, compared with normal saline-treated cells; ${ }^{*} \mathrm{p}<0.05$, compared with cisplatin-treated cells.

A

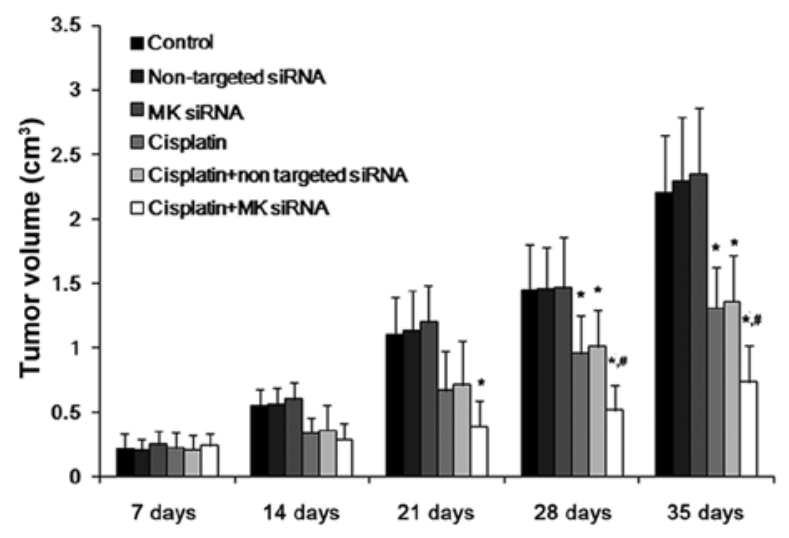

B

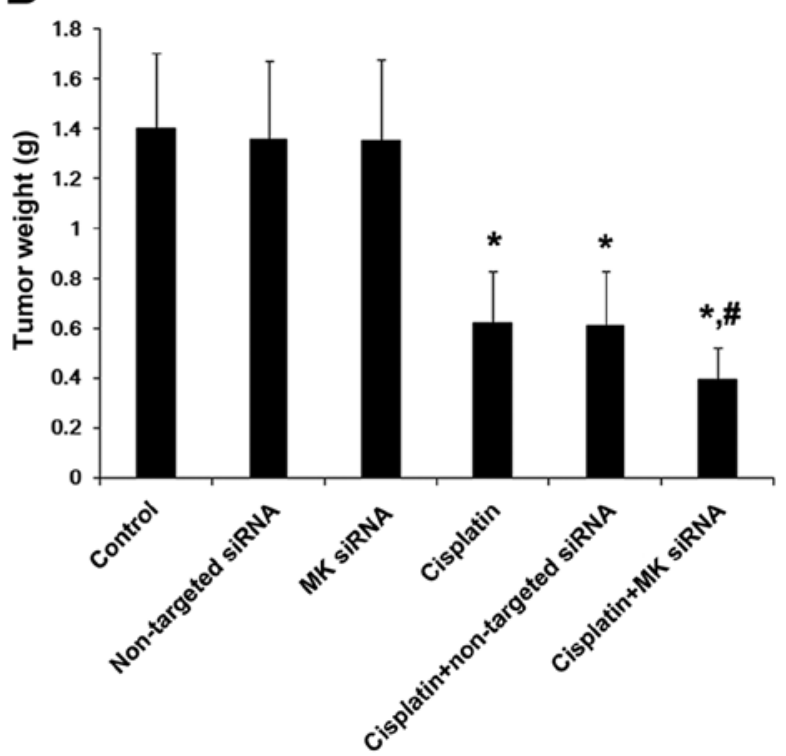

Figure 10. The inhibitory effect of the combined treatment with cisplatin and MK siRNA on the gowth of gastric cancer cells in nude mice. (A) Tumor volume changes in nude mice after cisplatin and MK siRNA treatment. (B) Tumor weights were measured 5 weeks after cisplatin and MK siRNA treatment. Data are expressed as mean $\pm \mathrm{SE}$. ${ }^{\mathrm{p}} \mathrm{p}<0.05$, compared with normal saline-treated cells; ${ }^{\#} \mathrm{p}<0.05$, compared with cisplatin-treated cells. 


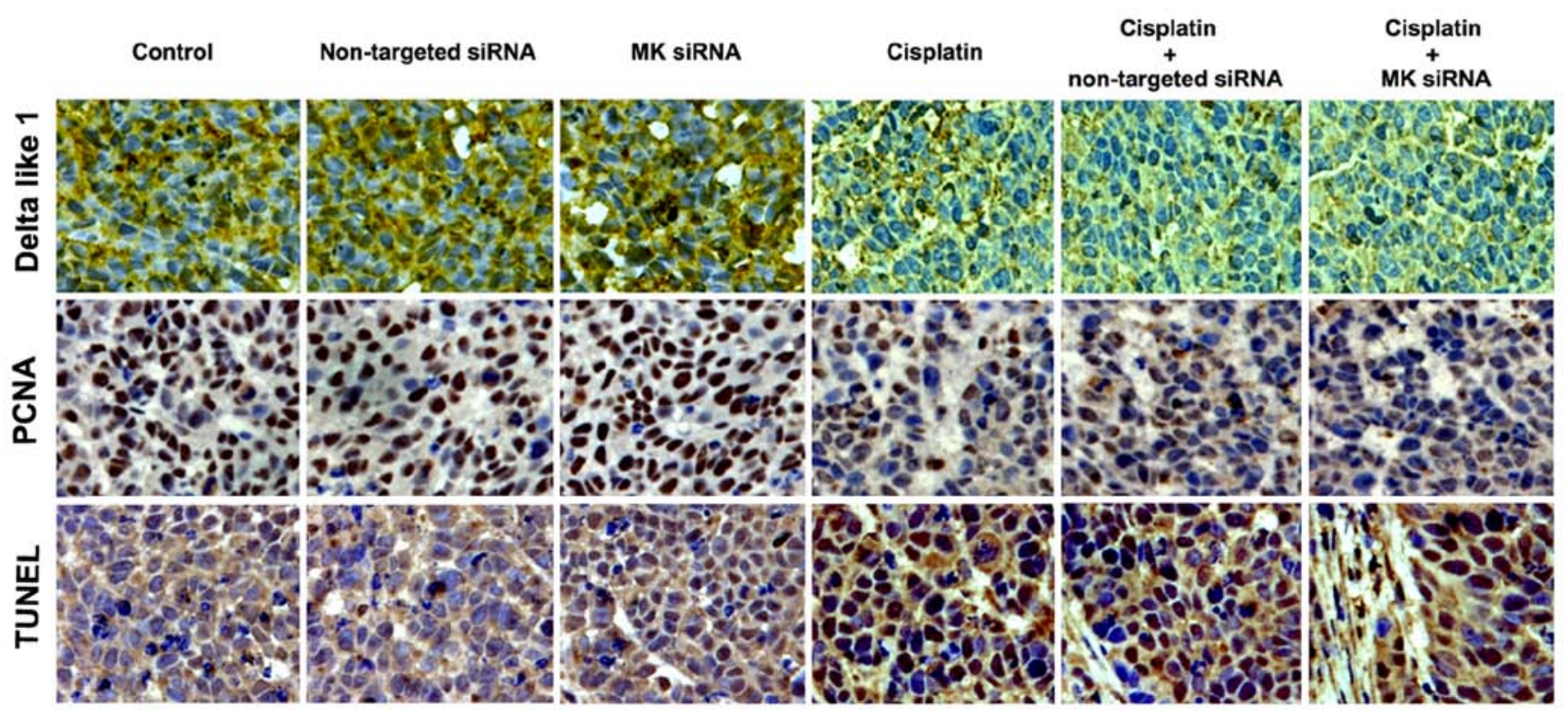

Figure 11. Representative images of immunohistochemical analysis of Delta-like 1 and PCNA and TUNEL analysis in the xenograft tumors of control, non-targeted siRNA, MK siRNA, cisplatin, cisplatin+non-targeted siRNA or cisplatin + MK siRNA.

A

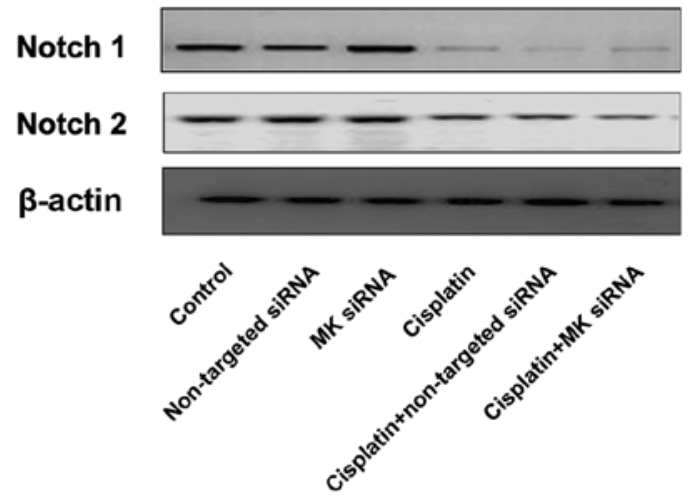

B

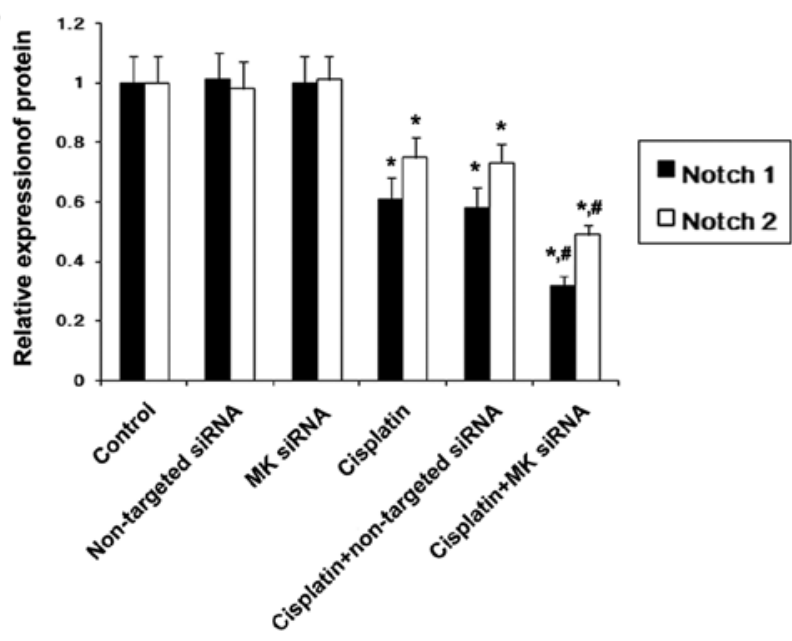

Figure 12. The effect of cisplatin and MK siRNA on the expression of Notch 1 and Notch 2 proteins in xenograft tumors. (A) Western blot analysis showed that the downregulation of Notch 1 and Notch 2 induced by cisplatin was strengthened by MK siRNA. (B) The relative expression of Notch 1 and Notch 2 protein. Data are expressed as mean $\pm \mathrm{SE}$. ${ }^{*} \mathrm{p}<0.05$, compared with normal saline-treated cells; ${ }^{*} \mathrm{p}<0.05$, compared with cisplatin-treated cells.

have shown that urinary MK and serum MK levels are elevated in cancer patients and are associated with disease progression. MK mRNA and protein levels are also reported to be both associated with the clinical stages and distant metastases in gastric cancer in Chinese patients $(12,18,19)$. Similarly in our study, MK protein expression was also found in gastric carcinoma tissues, and the extent of expression was correlated with the differentiation of tissue specimens. In cancer cell lines, high expressed MK protein was also obseverd in poorly differentiated cells. These results further proved that MK may be involved in the development of human gastric cancer. However, the possible mechanisms of MK in the pathogenesis and the therapeutic effect of MK in gastric cancer are still not fully clarified, which then prompted our further experiments.

As an oncoprotein that functions in gastric carcinogenesis, MK is reported to positively regulate the proliferation of human gastric cancer cells (14). Thus, we investigated the therapeutic effect of the recombinant human MK (rhMK) on AGS cells, the cells which could detect the highly expressed MK protein. In contrast to the results of Xu et al (14), rhMK did not show a proliferative effect on cancer cells in our study. However, the cytotoxic effect of cisplatin on AGS cells in vitro was attenuated by rhMK via reducing the cisplatin-induced apoptosis. The different selection of the cell line may lead to the different result, but on the other hand, our results would indicate that MK might be involved in the chemotherapyresistance in gastric cancer. Then, MK-targeted siRNA was applied to study the effect of MK inhibition on AGS cells. Although MK-targeted siRNA treatment did not play a growth-inhibitory function on AGS cells, it promoted the cytotoxic effect of cisplatin by inducing more severe apoptosis. Moreover, the tumor growth in nude mice was inflenced by 
MK inhibition as well. Previous study has also shown that the apoptosis of MK siRNA transfected cells might be mediated by the mitochondria-apoptosome-mediated pathway. The downregulated expression of $\mathrm{Bcl}-2$ and upregulated expression of Bax decreased mitochondrial membrane potential, and led to release of cytochrome $c$, and activated caspase-3, -8 and -9 , especially caspase-3 and -9 , induced apoptosis of the cells (20). These results suggest that MK inhibition may benefit chemotherapy treatment in gastric cancer.

Notch signaling is defined as an evolutionarily conserved local cell interaction mechanism that is involved in a variety of cellular processes. Recently, emerging evidence suggests that Notch signaling pathway is one of the most important signaling pathways in chemotherapeutic drug resistance. Importantly, interfering Notch signaling by $\gamma$-secretase inhibitors (GSIs) or downregulation of Notch 1 receptor can induce drug sensitivity, leading to increased inhibition of cancer cell growth and metastasis (21-25). The role of Notch signaling pathway in cancer cell drug resistance was widely studied, and studies have demonstrated that Notch signaling regulate the formation of cancer stem cells (CSCs) and contributes to epithelial-mesenchymal transition (EMT), which was found associated with drug resistance (26-28). It was also reported that inhibition of Notch signaling downregulates pro-survival pathways and anti-apoptosis genes. Previous studies have shown that the aberrant expression of Notch 1 and Notch 2 was related to a growing number of solid tumors, as well as gastric cancer $(29,30)$. The Notch signaling pathway is a conserved ligand-receptor signaling pathway present in most multicellular organisms. The mammalian canonical ligands are designated as Delta-like (Delta 1-4) or Serrate-like ligands known as Jagged 1 and Jagged 2, and the Notch receptors are single-pass transmembrane receptor proteins encoded by Notch genes (Notch 1-4) (31,32). In our study, the expression of Notch 1, Notch 2, Delta 1 and Jagged 1 was observed in control AGS cells. When interfering with MK-siRNA, these protein changes were significantly impacted in cisplatintreated cells. Many studies have focused on the fact that MK exerts an anti-apoptotic effect by influencing the Bcl-2 family proteins and the caspase cascade. In the present study, Notch signaling related proteins were obviously downregulated in MK hypoexpressing cells, along with the decrease of apoptotic cells. This result suggested that Notch signaling pathway could be suppressed by MK inhibition, which facilitated the chemotherapeutic effect of cisplatin in gastric cancer. Understanding the molecular mechanisms, driving MK-induced chemotherapeutic agent resistance, will provide benefits in developing new therapies for gastric cancer.

In conclusion, suppression of MK gene promoted the antitumoral effect of cisplatin on human gastric cell line AGS in vitro and in vivo. The downregulation of Notch signaling pathway-related proteins induced by suppressing MK gene is likely to be involved in modulating the cisplatin-induced apoptosis in gastric cancer cells.

\section{Acknowledgements}

This study was supported by the National Natrual Science Foundation of China (no. 81300375), Suzhou Municipal Science and Technology Development (SYS201333 and
SYS201461) and Specialized Clinical Research Project of the Department of Science and Technology, Jiangsu (BL2014046).

\section{References}

1. Zhao EH, Ling TL and Cao H: Current status of surgical treatment of gastric cancer in the era of minimally invasive surgery in China: Opportunity and challenge. Int J Surg 28: 45-50, 2016.

2. Yang L: Incidence and mortality of gastric cancer in China. World J Gastroenterol 12: 17-20, 2006.

3. Takayama S, Wakasugi T, Funahashi $\mathrm{H}$ and Takeyama H: Strategies for gastric cancer in the modern era. World J Gastrointest Oncol 2: 335-341, 2010

4. Bornschein J, Kandulski A, Selgrad M and Malfertheiner P: From gastric inflammation to gastric cancer. Dig Dis 28: 609-614, 2010.

5. Kadomatsu K, Tomomura M and Muramatsu T: cDNA cloning and sequencing of a new gene intensely expressed in early differentiation stages of embryonal carcinoma cells and in midgestation period of mouse embryogenesis. Biochem Biophys Res Commun 151: 1312-1318, 1988.

6. Garver RIJ Jr, Radford DM, Donis-Keller H, Wick MR and Milner PG: Midkine and pleiotrophin expression in normal and malignant breast tissue. Cancer 74: 1584-1590, 1994.

7. O'Brien T, Cranston D, Fuggle S, Bicknell R and Harris AL: The angiogenic factor midkine is expressed in bladder cancer, and overexpression correlates with a poor outcome in patients with invasive cancers. Cancer Res 56: 2515-2518, 1996.

8. Koide N, Hada H, Shinji T, Ujike K, Hirasaki S, Yumoto Y, Hanafusa T, Kadomatsu K, Muramatsu H, Muramatsu T, et al: Expression of the midkine gene in human hepatocellular carcinomas. Hepatogastroenterology 46: 3189-3196, 1999.

9. Ye C, Qi M, Fan QW, Ito K, Akiyama S, Kasai Y, Matsuyama M, Muramatsu T and Kadomatsu K: Expression of midkine in the early stage of carcinogenesis in human colorectal cancer. Br J Cancer 79: 179-184, 1999

10. Mishima K, Asai A, Kadomatsu K, Ino Y, Nomura K, Narita Y, Muramatsu T and Kirino T: Increased expression of midkine during the progression of human astrocytomas. Neurosci Lett 233: 29-32, 1997

11. Ikematsu S, Okamoto K, Yoshida Y, Oda M, SuganoNagano H, Ashida K, Kumai H, Kadomatsu K, Muramatsu H, Takashi Muramatsu and Sakuma S: High levels of urinary midkine in various cancer patients. Biochem Biophys Res Commun 306: 329-332, 2003

12. Obata Y, Kikuchi S, Lin Y, Yagyu K, Muramatsu T and Kumai H; Tokyo Research Group on Prevention of Gastric Cancer: Serum midkine concentrations and gastric cancer. Cancer Sci 96: 54-56, 2005.

13. Kadomatsu K, Hagihara M, Akhter S, Fan QW, Muramatsu H and Muramatsu T: Midkine induces the transformation of NIH3T3 cells. Br J Cancer 75: 354-359, 1997.

14. Xu Y, Qu X, Zhang X, Luo Y, Zhang Y, Luo Y, Hou K and Liu Y: Midkine positively regulates the proliferation of human gastric cancer cells. Cancer Lett 279: 137-144, 2009.

15. Yoon C, Cho SJ, Aksoy BA, Park DJ, Schultz N, Ryeom SW and Yoon SS: Chemotherapy resistance in diffuse-type gastric adenocarcinoma is mediated by RhoA activation in cancer stem-like cells. Clin Cancer Res 22: 971-983, 2016.

16. Lee HW, Kim SJ, Choi IJ, Song J and Chun KH: Targeting Notch signaling by $\gamma$-secretase inhibitor I enhances the cytotoxic effect of 5-FU in gastric cancer. Clin Exp Metastasis 32: 593-603, 2015.

17. Mei H, Yu L, Ji P, Yang J, Fang S, Guo W, Liu Y and Chen X: Doxorubicin activates the Notch signaling pathway in osteosarcoma. Oncol Lett 9: 2905-2909, 2015.

18. Huang Y, Cao G, Wang H, Wang Q and Hou Y: The expression and location of midkine in gastric carcinomas of Chinese patients. Cell Mol Immunol 4: 135-140, 2007.

19. Zhao ZQ, Yang S and Lu HS: Expression of midkine and vascular endothelial growth factor in gastric cancer and the association of high levels with poor prognosis and survival. Mol Med Rep 5: 415-419, 2012.

20. Wang Q, Huang Y, Ni Y, Wang H and Hou Y: siRNA targeting midkine inhibits gastric cancer cells growth and induces apoptosis involved caspase-3,8,9 activation and mitochondrial depolarization. J Biomed Sci 14: 783-795, 2007.

21. Leong KG and Karsan A: Recent insights into the role of Notch signaling in tumorigenesis. Blood 107: 2223-2233, 2006. 
22. South AP, Cho RJ and Aster JC: The double-edged sword of Notch signaling in cancer. Semin Cell Dev Biol 23: 458-464, 2012.

23. Koduru S, Kumar R, Srinivasan S, Evers MB and Damodaran C: Notch-1 inhibition by Withaferin-A: A therapeutic target against colon carcinogenesis. Mol Cancer Ther 9: 202-210, 2010.

24. Wang Z, Li Y, Ahmad A, Azmi AS, Banerjee S, Kong D and Sarkar FH: Targeting Notch signaling pathway to overcome drug resistance for cancer therapy. Biochim. Biophys Acta 1806: 258-267, 2010.

25. Miele L: Notch signaling. Clin Cancer Res 12: 1074-1079, 2006.

26. Ding Y and Shen Y: Notch increased vitronection adhesion protects myeloma cells from drug induced apoptosis. Biochem Biophys Res Commun 467: 717-722, 2015.

27. Hang Q, Sun R, Jiang C and Li Y: Notch 1 promotes cisplatinresistant gastric cancer formation by upregulating lncRNA AK022798 expression. Anticancer Drugs 26: 632-640, 2015.
28. Zhao J, Nie Y, Wang H and Lin Y: miR-181a suppresses autophagy and sensitizes gastric cancer cells to cisplatin. Gene 576: 828-833, 2016.

29. Piazzi G, Fini L, Selgrad M, Garcia M, Daoud Y, Wex T, Malfertheiner P, Gasbarrini A, Romano M, Meyer RL, et al: Epigenetic regulation of Delta-Like1 controls Notch1 activation in gastric cancer. Oncotarget 2: 1291-1301, 2011.

30. Yeh TS, Wu CW, Hsu KW, Liao WJ, Yang MC, Li AF, Wang AM, Kuo ML and Chi CW: The activated Notch 1 signal pathway is associated with gastric cancer progression through cyclooxygenase-2. Cancer Res 69: 5039-5048, 2009.

31. Kopan R and Ilagan MX: The canonical Notch signaling pathway: Unfolding the activation mechanism. Cell 137: 216-233, 2009.

32. Artavanis-Tsakonas S, Rand MD and Lake RJ: Notch signaling: Cell fate control and signal integration in development. Science 284: 770-776, 1999. 